\title{
Provider Use of a Novel EHR display in the Pediatric Intensive Care Unit
}

\section{Large Customizable Interactive Monitor (LCIM)}

Onur Asan; Richard J. Holden²; Kathryn E Flynn; Yushi Yang ${ }^{3}$; Laila Azam; Matthew C Scanlon ${ }^{5}$

${ }^{1}$ Center for Patient Care and Outcomes Research, Division of General Internal Medicine, Department of Medicine, Medical College of Wisconsin, Milwaukee, WI,USA;

${ }^{2}$ School of Informatics and Computing, Indiana University-Purdue University, Indianapolis, IN, USA;

${ }^{3}$ Department of Industrial Engineering, University of Wisconsin-Madison, Madison, WI, USA;

${ }^{4}$ Department of Public and Community Health, Institute for Health and Society, Medical College of Wisconsin, Milwaukee, WI, USA;

${ }^{5}$ Department of Pediatrics, Division of Critical Care, Medical College of Wisconsin, Milwaukee, WI, USA

\section{Keywords}

Health information technology, pediatric intensive care units, workflow, clinical information systems/testing and evaluation

\section{Summary}

Objectives: The purpose of this study was to explore providers' perspectives on the use of a novel technology, "Large Customizable Interactive Monitor" (LCIM), a novel application of the electronic health record system implemented in a Pediatric Intensive Care Unit.

Methods: We employed a qualitative approach to collect and analyze data from pediatric intensive care physicians, pediatric nurse practitioners, and acute care specialists. Using semi-structured interviews, we collected data from January to April, 2015. The research team analyzed the transcripts using an iterative coding method to identify common themes.

Results: Study results highlight contextual data on providers' use routines of the LCIM. Findings from thirty six interviews were classified into three groups: 1) providers' familiarity with the LCIM; 2) providers' use routines (i.e. when and how they use it); and 3) reasons why they use or do not use it.

Conclusion: It is important to conduct baseline studies of the use of novel technologies. The importance of training and orientation affects the adoption and use patterns of this new technology. This study is notable for being the first to investigate a LCIM system, a next generation system implemented in the pediatric critical care setting. Our study revealed this next generation HIT might have great potential for family-centered rounds, team education during rounds, and family education/engagement in their child's health in the patient room. This study also highlights the effect of training and orientation on the adoption patterns of new technology.

\section{Correspondence to:}

Onur Asan, PhD

Center for Patient Care and Outcomes Research Division of General Internal Medicine, Department of Medicine

Medical College of Wisconsin

Milwaukee, WI 53226

Phone: +14149558815

Fax: +14149556689

Email: oasan@mcw.edu
Appl Clin Inform 2016; 7: 682-692

http://dx.doi.org/10.4338/ACl-2016-02-RA-0030 received: February 29, 2016

accepted: June 14, 2016

published: July 20, 2016

Citation: Asan O, Holden RJ, Flynn KE, Yang Y, Azam L, Scanlon MC. Provider use of a novel EHR display in the pediatric intensive care unit: Large customizable interactive monitor (LCIM). Appl Clin Inform 2016; 7: 682-692 http://dx.doi.org/10.4338/ACI-2016-02-RA-0030 Funding

Financial support was provided by the Agency for Healthcare Research and Quality (Grant \# 1R21HS023626-01) for this study. 


\section{Introduction}

Health Information Technology (HIT) adoption and implementation have increased at a rapid pace since the Health Information Technology for Economic and Clinical Health (HITECH) Act was passed in 2009 [1-4]. Some studies have shown that HIT has the potential to improve overall care and patient engagement, the latter a particular "hot topic" in recent healthcare research [5-7]. However, the potential of some technologies have not been realized. Simply implementing a technology does not guarantee its use $[8,9]$. Regardless of the purported quality of the technology or the fact that a given HIT was installed, ultimately, it is the perceptions of those intended to use the technology that determine both its use and whether potential benefits are realized [10, 11].

Studies have shown that even when clinicians recognize the value of HIT, they may report experiencing its undesirable impact on communication, workflow, and patient care [12-15]. Various steps to decrease such problems and increase successful adoption and acceptance of HIT have been described, such as understanding the needs of providers during the design process [16, 17], designing and testing for usability $[18,19]$ and providing adequate training for those who are supposed to use it [20]. These and other steps could enhance the perceived usefulness and ease of use of HIT, the two factors most correlated with end-user acceptance, i.e. satisfaction and intent to use it in the future [21].

The adoption and meaningful use of electronic health records systems (EHRs) has been reported as one of the major priorities for improving the quality and efficiency of the healthcare system [4], however the relationship between how EHRs are used by providers and healthcare quality remains inconsistent $[22,23]$. It is essential to explore and understand use routines, variations in use behaviors, characteristics, and functionalities of EHRs when implemented. Some studies explored the individual physician-level differences in EHR use patterns and identified the relationship between EHR use patterns and some measures, such as physicians' perceived uncertainty, satisfaction, usefulness and information needs [23, 24]. Several studies have focused on the use of EHRs/HIT and described the nature and functions of EHR system use after the implementation and adoption of the system in various settings. For instance, the use patterns of EHR functionalities have been explored in primary care settings [25-28], psychology clinics [29], and inpatient settings [4, 30]. One study also explored the relationship between providers' comfort with using computers and the number of EHR features used [31]. In addition to mandated EHRs, there have been studies focusing on the design and use of novel applications of HITs that are not mandated, such as electronic white boards [32], tablets [33], and mobile health applications [34, 35]. These novel systems have the potential to enhance consumer engagement, and it is essential to understand the use of such technologies in order to maximize their usefulness and contribute to future design improvements.

In this study, we focus on a novel HIT application that has the potential to become a next generation system, but to our knowledge has no previous IT research published in the literature. This is a Large Customizable Interactive Monitor (LCIM), a 42" inch (diagonal) flat panel touch screen monitor that displays validated patient information including vital signs, laboratory results, medications and interventions recorded in the EHR. The LCIM is located in each patient room. In contrast to traditional HIT that requires a secure log-in by providers, the LCIM can be used by providers as well as patients and their families without repeated login. The purpose of this qualitative study, which is a part of a larger mixed-methods study, was to understand providers' baseline use as well as use characteristics and routines of the LCIM technology in the PICU.

\section{Research Methods}

\subsection{Overview}

We used qualitative semi-structured, in-person interviews to collect data from critical care providers in the PICU, including physicians, pediatric nurse practitioners and acute care specialists. We collected contextual data on providers' use of the LCIM, which was installed in November 2012. Interviews were performed January to April, 2015. The study hospital's institutional review board approved this study. 


\subsection{Setting}

The setting for the study was the PICU of a freestanding children's hospital in a mid-sized Midwestern city. The PICU is a 72-bed unit comprised of three 24-bed floors, which provides highly specialized care to critically ill children and neonates and admits over 3000 patients a year. The first floor primarily admits children that have congenital heart defects. Services provided include monitoring of assist devices, extracorporeal membrane oxygenation, post-heart surgery and post-organ transplant. The second floor is a mix of surgical and medical patients, such as body trauma, transplants and innovative surgical procedures. The third floor also is a mix of surgical and medical patients but focus on children with medical life threatening illnesses such as sepsis, burns and oncologic disorders. Each patient room in the PICU was equipped with a wall mounted LCIM at the time of the implementation of a new commercial EHR. Informal observations (by YY) indicated a wide variety of actual use of the LCIM by providers.

\subsection{Technology}

The study hospital implemented a commercially available EHR system in November of 2012. The EHR was in use in all inpatient environments, the emergency department, the operating room environment (including anesthesia services), and health system associated primary and subspecialty ambulatory care settings. As a part of the implementation package, PICU management decided to install the LCIM in each PICU patient room. This LCIM installation was the third in the U.S. and the first in a pediatric setting. The LCIM is an interactive "view only" technology with no ability to enter data. The LCIM technology utilized the Epic Monitor (v 2010, Epic Systems Corporation, Verona, WI). The institution ensured that the LCIM was HIPAA compliant.

The LCIM is an additional software tool that works with the Epic EHR and allows the creation of customized review widgets for display of a patient's chart. The principal use of the tool is to display information about one patient on a large touch-screen monitor near the patient. The customizable nature of the software has the potential to display data from multiple patients, whether as a "tracking board" or in an overview of a patient care unit. While the tool is limited to display only those elements available in the EHR, the nature of the LCIM software creates additional limitations in which patient data can be displayed and in what manner. As a result, our evaluation reflects the use of the LCIM as an interactive window to the EHR.

At the time of observations and data collection, data viewable using the LCIM included discrete and trend vital sign information validated by bedside nurses; the patient problem list; lab test results; a list of medications and infusions with doses, routes, frequency and last administration time; documented fluid "ins and outs; ventilator settings; and all indwelling lines, drains and airways with date/ time of placement. Finally, there was no specific LCIM training or orientation for providers during the Epic implementation.

\subsection{Recruitment Process and Participants}

During the data collection period, potential participants of the study were 39 critical care medical providers working in the PICU. Recruitment flyers were posted in the units with the contact information of the research coordinator. The research coordinator attended several provider meetings to announce the study and encourage participation. Providers who wished to participate voluntarily contacted the research coordinator for an interview appointment. Interviews were completed with 36 participants out of the 39 (92\%) providers in the unit, of whom, 19 were attending physicians, 16 were pediatric nurse practitioners, and one was an acute care specialist. Most participants were white, non-Hispanic (97\%). Four (11\%) of the participants were between 18-29 years old, 13 (36\%) between 30-39, 11 (31\%) between 40-49, 7 (19\%) between 50-59, and one (3\%) was older than 60 years. Participants' average tenure at the hospital was 17 years (mean), and average tenure in their current role was 9 years (mean). Additionally, participants had an average 8 years' experience with EHR/CPOE systems, and specifically using the Epic system of the hospital for approximately 3 years (mean). 


\subsection{Interviews}

The individual interview sessions were conducted in a private office in the PICU by the first author (OA). We used a semi-structured guide that served as a checklist of topics to explore during the interview. The interview guide was developed by the study team based on specific study objectives and following the Technology Acceptance Model (TAM) [36]. It was also informed by 10 hours of pilot observations of the use routines conducted in the PICU (YY). The interview guide included questions regarding physicians use routines, how and when they use the LCIM, training and familiarity as well as perceived usefulness and ease of use questions ( $>$ Table 1). The interview guide was reviewed by two Human Factors Engineers $(\mathrm{OA}, \mathrm{RH})$ and one methodologist $(\mathrm{KF})$. It was then piloted with a pediatric intensivist (MS) to test: a) whether the questions in the interview guide were clear and understandable; b) whether interviewees would interpret the questions in a manner consistent with the researcher's conceptualizations; c) that the right healthcare terminology was used; and d) the appropriateness of probes or need for additional probes. The interview guide was revised based on the feedback obtained in the pilot interview.

Interviews were audiotaped and transcribed verbatim for analysis. Each interview lasted 20-35 minutes $($ mean $=24$ minutes). Four researchers $(\mathrm{OA}, \mathrm{LA}, \mathrm{YY}, \mathrm{KF})$ met after reading eight interview transcripts to discuss initial themes and definitions of each theme. A coding book was developed to guide the coding process [37]. We used NVivo 10 to facilitate coding and analyses [38]. The first round of thematic coding process was completed by two independent coders (LA, YY). A third independent coder (OA) served as a judge to adjudicate any disagreements. Reliability checks were conducted throughout the coding process and the two main coders achieved acceptable Cohen's Kappa inter-coder reliability of higher than 0.85 . Then, a second round of coding was completed using a condensed meaning analysis for each quote coded by the two coders. Appropriate revisions of the themes and codes were made until consensus was achieved between the two coders. The third independent coder reviewed all the condensed codes, adjusted any discrepancies, and categorized the codes into a sociotechnical systems model. Finally, we held a group meeting with the addition of a human factors expert (RH) to discuss emergent themes and develop the reports.

\section{Results}

The findings from the thematic analysis were classified into three groups; a) providers' familiarity with the LCIM, b) providers' use routines, i.e., when and how they use the LCIM, and c) reasons why they use or do not use the LCIM.

\subsection{Providers' familiarity with the LCIM}

Providers' familiarity with the LCIM varied widely and appeared to be affected by a perceived lack of training. Most providers stated there was no formal training on how to use the LCIM or its features, functions, reason for implementation, and purpose. Some providers reported being very familiar with the LCIM and its features. These providers reported becoming familiarized on their own or learning from other users, as demonstrated by this comment,

"It is a touch screen. I just played with it, I've watched other people use them."

All providers stated they were aware of the LCIM, but it was clear providers did not know all the existing content and features. We categorized providers into three groups based on familiarity obtained from their responses: high (20\%), moderate (60\%), and low/no (20\%). High-familiarity providers reported knowing most, though clearly not all, the available functions and features. A comment typical of providers in this group was,

"I am very familiar with it, I've been using it since we installed them, there might be things I don't know about it but I know most of the functionality of it". 
Moderate-familiarity providers are aware of some specific features or content and wanted to learn more about other specific functions or features available, such as,

"I don't know if a consult's note is there. I'm guessing it probably is, but I have no idea how to access that".

Low/no-familiarity providers often mentioned lack of training, i.e.,

"I am not familiar. I have never been shown how to use it and so do not know the functionalities and features on that".

Across all three groups, providers were unfamiliar with certain features, for example, how to power up a turned-off LCIM.

\subsection{Providers' use routines - how and when they use the LCIM}

Table 2 lists different major ways of using the LCIM and situations in which providers reported using it. We found three main uses: 1) Using the LCIM as a snapshot of the current data, that is, gazing at it to view overall data; 2) Touching and interacting with the screen to get more detailed data, perhaps zooming in on a specific section; and 3) Using it as a teaching tool for families or other providers. Common situations when the LCIM was used were: 1) During rounds,

"At minimum, I use it at the time that we round during the pre-rounding, rounding with the team, and then when we do sign out rounds";

2) When providers walk in the patient room,

"I use it as a snapshot as I walk into the room. First of all just to remind me of the patient name and age and because sometimes you forget walking in - and then as a snapshot of some of the things going on in the last bit of time, like the vital signs and the urine output";

and 3) When interacting with a family member or a team member,

"If I think there's an interesting point to either show the house staff or families. It's a very big projection screen and I can like show, " $X$ ' happened and here you see the response of what happened" using it more as a teaching tool than as a rounding tool".

In addition to Table 2, some providers also reported some specific use characteristics. A few providers reported that they use LCIM when they assess a concern for the patients' health. It was also reported that they use LCIM as a reference point rather than computer desktop when they respond specific questions from families. Further, several providers reported using LCIM more often if the patient has severe illness and the parents are more computer savvy. In addition, providers who are on-call for the night expressed a benefit of using the LCIM to get very quick overview once they are called to a room.

\subsection{Reasons for using or not using the LCIM}

When asked why providers used the LCIM, common categories of answers were: a) It is there (simply the existence of LCIM in the room is listed as a reason to use it); b) It is readily available as a quick reference point; and c) they do not need to log into the computer to access data, they can just walk in and glance at it. One provider mentioned all three reasons in their answer:

"because it is there, it's very accessible. It's easy to get the data without logging into the computer. If I'm with a group of people it's easy to look at the information together. Visually it's far more appealing than the computer." 
We obtained several reasons from non-user providers regarding why they did not use it. The most common reason was lack of familiarity with the LCIM and feeling more comfortable with using a desktop computer instead, e.g.

"I think because I'm not that familiar with it $<\mathrm{LCIM}>$ and I am more familiar with how to find the information on a computer and that's more comfortable for me so that's what I do".

A few providers thought that LCIM was not embedded in their workflow

"I could do without it because I have done without it. I'm not used to using it you know. It hasn't been embedded in my brain to use enough to say I really need that".

Other reported reasons were: 1) Not knowing what type of information the LCIM provides; 2) Preferring to use Computers on the Wheels (COWs) and rounding outside of the room in the hall; 3 ) Finding it easier to use other technologies to get data some specific visual data, such as in room vital monitors. As one provider described,

"My work flow is different. My workflow - I get here in the morning and I open the charts of the patients and I do a quick view of what I want to review very quick. And so whatever is on the LCIM, I usually get it from myself opening the charts. And during rounds I don't need them either because as I do rounds I get information from whoever is presenting the data, but again doesn't work in my workflow".

\subsection{Contents used and not used in LCIM}

Providers who were moderate or high users also reported the most and least commonly used features. Most respondents reported the two most commonly used features are viewing lab results, and I's and O's (Ins and outs). More than half of the providers also stated they use vital trends. The rest of the features/contents used at some degrees are ventilator settings, medication list, problem list, weight for dosage, and respirator for children. On the other hand, three providers reported using only the lab values features. Several providers reported specific contents they did not use including respiratory components, drops, medication list and vital trends. Some providers preferred to use desktop computers over the LCIM for certain contents:

"Well, probably the data I gravitate to the most include the I's and O's, the weight, the lab results, I don't use the respiratory component, vital sign trends I use but not to the degree I would go to the computer for, it's a little bit too crude of a trend data, and medications", or as another provider reported "I usually don't use it for ventilator settings because I just look at the ventilator".

\section{Discussion}

There is increasing attention to the role of HIT in patient-centered, high quality care. While the literature on how EHR systems affect quality and patient-centered care has grown substantially, this is not the case for so-called novel, next generation HIT systems. Implementing a next generation technology can be accompanied by unintended consequences, unforeseen use patterns, and various implementation challenges that influence system use and usefulness $[18,20]$. Therefore, it is important to conduct baseline studies of the use of such technologies after implementation. This study is notable for being the first to investigate a LCIM system, a next generation system implemented in the pediatric critical care setting.

First, our results showed a general lack of familiarity with the system among providers, compounded by the lack of formal training, which meant learning was accomplished by self-exploration or observing colleagues using the system. Some reported not even knowing the purpose of the system. Lack of meaningful or systematic training for a newly impelemnted HIT/EHR was reported by several other studies in the literature $[18,20]$. Organizations generally have a $2-3$ hour one-time training session on EHRs, but most users are not trained on how to incorporate the technology into 
their workflow, patient visits, and communication with patients or families $[39,40]$. In this study, several providers reported lack of training as the main reason for low or no use of the LCIM. Training is reported elsewhere as a factor that impacts actual use [41] so a better and meaningful training may increase the initial use of such novel technologies when implemented. Given the lack of systematic training, it was not surprising to find that providers had various familiarity levels with the LCIM. Indeed, most stated that they likely did not know all of the available functions. This also indicates lost opportunity for providers to realize all advertised benefits of this HIT.

The second main emerging theme is providers' LCIM use routines. Our study revealed that the LCIM might have great potential for family-centered rounds, team education during rounds, and family education about and engagement in their child's health. Providers reported mostly using the LCIM during rounds as a teaching tool, since it provides a large-screen overview of useful data that is simultaneously visible to multiple people. Family-centered rounds have been recommended by recent studies due to several benefits, including their positive impact on family engagement $[42,43]$. Having families available during rounds and visually showing them the values using the LCIM may be a key part of future family-centered rounds and might improve family engagement. Furthermore, there has been significant attention to the family-centered health care model with an appropriate focus on family engagement and patient-provider communication (often parent-provider communication in pediatrics) $[44,45]$. Optimal communication strategies are essential for a successful provider-parent relationship and effective exchange of information and understanding of care. The use of the LCIM as a tool to communicate and educate parents in the patient room, as was reported by some of our providers, might be an effective way to empower families and improve their understanding of the care.

Third, the needs of providers are likely related to easy and quick "just-in-time" access to information needed for clinical care [32]. This is particularly true in the ICU setting, since it is critical to access timely information to have a better assessment of patients [46]. The main reason why providers reported using the LCIM was indeed "just in time" access to information. One of the main advantages of the LCIM is the fact no password is required for use, unlike traditional HIT. Furthermore, being able to have a snapshot of patient status in one view was reported as a reason to use LCIM. Of the available features, providers were most likely to access lab results and I's and O's, not surprising, as both functions give a quick overall snapshot of the patient's status that is very critical in ICU settings. The system generates nine widgets so nine different data types can be displayed at once on the screen, giving an overview of the patient when providers walk into the room. This design, having readily available information and a snapshot of the patient's current status, would have implications for providers continuous awareness regarding patient's status. It may also facilitate the anticipation of future patient needs and clinical activities, a function necessary for bedside pediatric intensive care [17].

Fourth, our results reveal some reasons for providers not using the LCIM, including lack of training and lack of familiarity. In addition, some providers thought that the LCIM was not embedded in their workflow. Previous studies also showed this as a reason to resist using newly implemented technologies [40]. Furthermore, providers stated they use other alternative technologies instead of the LCIM in the room, especially for some specific functions, such as using COWs during the rounds or the in-room vitals monitor to look at the vital trends in detail.

Lastly, one of the main concern regarding this technology is privacy and security due to the no $\log$ in requirement. Each of the 24-bed floors in the PICU is a locked unit with control of all visitors and staff. Beyond the security of the PICU, each patient room is a private room with the LCIM monitor displayed within the privacy of the room. As the only people accessing a patient's room (and thus potentially able to view data) are: 1) family members, 2) direct care providers, and/or 3) invited guests of the family. There is no more than minimal risk of a random person seeing protected health information. While there is the potential to display trend lines of information such as heart rate over time, the individual data values are too small to be viewed from any distance without entering the patient room and approaching the mounted display.

This study had both strengths and weaknesses. We had a very high response rate (90\%). Thus maximizes variability in reported experiences and perceptions allowing a relatively large sample size for an interview study. However, it investigated a single pediatric hospital and therefore the results may have limited generalizability. Since our the findings are consistent with studies of other technol- 
ogies, we suspect similar implementations in other settings may result in similar findings. Related to this, because the setting was a 72-bed ICU that had a dedicated cardiac, surgical and medical floors, the broad range of patient populations in this single ICU may result in findings that would apply to other organ center specific ICUs, even perhaps in the adult setting. The interviews occurred 2 years after the implementation due to the funding issues, however it would be better to assess such technologies within the first year of implementation. Finally, we urge additional studies to investigate the use of this technology and similar technologies, including longitudinal, quantitative, objective assessment and patterns of use.

\section{Conclusions}

This study contributes to an understanding of providers' use characteristics of a novel, next generation HIT systems in pediatric critical care. Our findings demonstrate that providers' style of use as well as use characteristics of this novel HIT may vary for several reasons. A main message is to understand the importance of training on and orientation for this type of novel technology regarding its goal, purpose of implementation, functions and potential contribution to patient care. These findings can also be used to improve understanding of physicians' information needs in the context of novel HIT-enabled healthcare delivery.

\section{Clinical Relevance Statement}

Implementing a next generation health information technology is accompanied by unintended consequences, unforeseen use patterns, and various implementation challenges that influence system use and usefulness. It is therefore important to conduct baseline studies of the use of such technologies as soon as they are implemented. This study is notable for being the first to investigate a LCIM system, a next generation system implemented in the pediatric critical care setting.

\section{Conflict of Interest}

The authors declare that they have no conflicts of interest in the research.

\section{Human Subjects Protections}

This study was approved by Children Hospital of Wisconsin's institutional Review Board.

\section{Acknowledgements}

We acknowledge the financial support provided by the Agency for Healthcare Research and Quality (Grant \# 1R21HS023626-01) for this study. This study would not have been possible without leadership team support. We thank Kathy Murkowski for arranging the interviews with PICU doctors for us. 
Table 1 Sample interview questions

How familiar are you with the "Large Epic Monitor" in the ICU?

Do you use the "Large Epic Monitor" while working in the PICU?

If yes, when and how frequently do you use it?

If yes, how do you use it? What information do you use it to access?

If yes, why do you use it?

If no, what prevents you from using it? (or why not?)

How do you use the Large Epic Monitor with families?

Do you know what type of information, functionalities and features the "Large Epic Monitor" provide?

If yes, please list them

If no, why did not you feel the need to learn it

Did you receive any training for using the "Large Epic Monitor" in ICU rooms?

If yes, tell me about the training you received.

If no, how did you learn to use it?

Table 2 Providers' LCIM Use Routines (when and how they use it)

\begin{tabular}{|l|l|}
\hline Theme & Situations of the use (\# of participants mentioning) \\
\hline Source of Information & $\begin{array}{l}\text { - To get a summary of patient's status and progress (11) } \\
\text { - To access specific lab or vital results (11) }\end{array}$ \\
\hline Interaction with families & $\begin{array}{l}\text { - To address patient families' specific questions (8) } \\
\text { - To proactively scroll through it and share information with the family (7) } \\
\text { - To educate families using labs, I's and O's, vital trends and graphs and interpret } \\
\text { the data (10) }\end{array}$ \\
\hline Team Communication/ & $\begin{array}{l}\text { - To use for information sharing and discussion points between providers (17) } \\
\text { - To educate medical students/interns (4) }\end{array}$ \\
\hline Use times/frequencies & $\begin{array}{l}\text { - During the rounds (21) } \\
\text { - During pre-rounds (5) } \\
\text { - A couple of times during the day (9) } \\
\text { - Minimum/no use (9) } \\
\text { - Avoiding use during the night (4) } \\
\text { - Using LCIM if other computers are occupied in the room (4) }\end{array}$ \\
\hline
\end{tabular}




\section{References}

1. Shoolin JS. Change management-Recommendations for successful electronic medical records implementation. Appl Clin Inform 2010; 1(3): 286-292.

2. Messeri P, Khan S, Millery M, Campbell A, Merrill J, Shih S, Kukafka R. An information systems model of the determinants of electronic health record use. Appl Clin Inform 2013; 4(2): 185-200.

3. Blumenthal, D. Stimulating the adoption of health information technology. N Engl J Med 2009; 360(15): 1477-1479.

4. Jha AK, DesRoches CM, Campbell EG, Donelan K, Rao SR, Ferris TG, Shields A, Rosenbaum S, Blumenthal D. Use of electronic health records in US hospitals. N Engl J Med 2009; 360(16): 1628-1638.

5. Hayrinen K, Saranto K, Nykanen P. Definition, structure, content, use and impacts of electronic health records: a review of the research literature. Int J Med Inform 2008; 77(5): 291-304.

6. Sheikh A, Sood HS, Bates DW. Leveraging health information technology to achieve the "triple aim" of healthcare reform. J Am Med Inform Assoc 2015; Jul 22 (4): 849-856.

7. White A, Danis M. Enhancing patient-centered communication and collaboration by using the electronic health record in the examination room. JAMA 2013; 309(22): 2327-2328.

8. Karsh BT, Weineger MB, Abbott PA, Wears RL. Health information technology: fallacies and sober realities. J Am Med Inform Assoc 2010; 17(6): 617-623.

9. Holden R, Karsh BT. A theoretical model of health information technology usage behaviour with implications for patient safety. Behav Inf Technol 2009; 28(1): 21-38.

10. Holden RJ. Physicians' beliefs about using EMR and CPOE: in pursuit of a contextualized understanding of health IT use behavior. Int J Med Inform 2010; 79(2): 71-80.

11.Delone WH, McLean ER. The DeLone and McLean model of information systems success: a ten-year update. J Manag Infom Syst 2003; 19(4): 9-30.

12. Harrison MI, Koppel R, Bar-Lev S. Unintended consequences of information technologies in health care-an interactive sociotechnical analysis. J Am Med Inform Assoc 2007; 14(5): 542-549.

13. Carayon P, Wetterneck TB, Alyousef B, Brown RL, Cartmill RS, McGuire K, Hoonakker PL, Slagle J, Van Roy KS, Walker JM, Weinger MB. Impact of electronic health record technology on the work and workflow of physicians in the intensive care unit. Int J Med Inform 2015; 84(8): 578-594.

14. Grabenbauer L, Skinner A, Windle J. Electronic health record adoption - maybe it's not about the money: physician super-users, electronic health records and patient care. Appl Clin Inform 2011; 2(4): 460-471.

15. Hanauer D, Zheng K. Measuring the impact of health information technology. Appl Clin Inform 2012; 3(3): 334-336.

16. Rogers M, Zach L, An Y, Dalrymple P. Capturing information needs of care providers to support knowledge sharing and distributed decision making. Appl Clin Inform 2012; 3(1): 1-13.

17. Holden RJ, Rivera-Rodriguez AJ, Faye H, Scanlon MC, Karsh BT. Automation and adaptation: nurses' problem-solving behavior following the implementation of bar-coded medication administration technology. Cogn Technol Work 2013; 15(3): 283-296.

18. Fossum M, Ehnfors M, Fruhling A, Ehrenberg A. An evaluation of the usability of a computerized decision support system for nursing homes. Appl Clin Inform 2011; 2(4): 420-436.

19. Middleton B, Bloomrosen M, Dente MA, Hashmat B, Koppel R, Overhage JM, Payne TH, Rosenbloom ST, Weaver C, Zhang J. Enhancing patient safety and quality of care by improving the usability of electronic health record systems: recommendations from AMIA. J Am Med Inform Assoc 2013; 20(e1): e2-e8.

20.Duke P, Frankel RM, Reis S. How to integrate the electronic health record and patient-centered communication into the medical visit: a skills-based approach. Teach Learn Med 2013; 25(4): 358-365.

21. Hoonakker PL, Carayon P, Walker JM. Measurement of CPOE end-user satisfaction among ICU physicians and nurses. Appl Clin Inform 2010; 1(3): 268-285.

22. Stead WW, Lin H. Computational technology for effective health care: immediate steps and strategic directions. 2009: Natl Academy Pr.

23.Lanham HJ, Sittig DF, Leykum LK, Parchman ML, Pugh JA, McDaniel RR. Understanding differences in electronic health record (EHR) use: linking individual physicians' perceptions of uncertainty and EHR use patterns in ambulatory care. J Am Med Inform 2014; 21(1): 73-81.

24. Makam AN, Lanham HJ, Batchelor K, Moran B, Howell囚Stampley T, Kirk L, Cherukuri M, Samal L, Santini N, Leykum LK, Halm EA. The good, the bad and the early adopters: providers' attitudes about a common, commercial EHR. J Eval Clin Pract 2014; 1: 36-42.

25. Rao SR, Desroches CM, Donelan K, Campbell EG, Miralles PD, Jha AK. Electronic health records in small physician practices: availability, use, and perceived benefits. J Am Med Inform Assoc 2011; 18(3): 271.

26. Fiks AG, Alessandrini EA, Forrest CB, Khan S, Localio AR, Gerber A. Electronic medical record use in pediatric primary care. J Am Med Inform Assoc 2011; 18(1): 38-44. 
27. Krist AH, Beasley JW, Crosson JC, Kibbe DC, Klinkman MS, Lehmann CU, Fox CH, Mitchell JM, Mold JW, Pace WD, Peterson KA. Electronic health record functionality needed to better support primary care. J Am Med Inform 2014; 21(5): 764-771.

28. Dufendach KR, Eichenberger JA, McPheeters ML, Temple MW, Bhatia HL, Alrifai MW, Potter SA, Weinberg ST, Johnson KB, Lehmann CU. Core Functionality in Pediatric Electronic Health Records. 2015.

29. Cellucci LW, Cellucci T, Stanton M, Kerrigan D, Madrake M. Current status and future directions of EMR use in psychology clinics. Health Policy and Technology 2015; 4(2): 91-99.

30.Lehmann CU, Weinberg ST, Alexander GM, Beyer EL, Del Beccaro MA, Francis AB, Handler EG, Johnson TD, Kirkendall ES, Lighter DE, Morgan, SJ. Pediatric Aspects of Inpatient Health Information Technology Systems. Pediatrics 2015; 135(3): e756-e768.

31. Bishop TF, Ryan MS, McCullough CM, Shih SC, Casalino LP, Ryan AM. Do provider attitudes about electronic health records predict future electronic health record use? Healthc (Amst) 2015; 3(1): 5-11.

32. Wong HJ, Caesar M, Bandali S, Agnew J, Abrams H. Electronic inpatient whiteboards: Improving multidisciplinary communication and coordination of care. Int J Med Inform 2009; 78(4): 239-247.

33. Vawdrey DK, Wilcox LG, Collins SA, Bakken S, Feiner S, Boyer A, Restaino SW. A tablet computer application for patients to participate in their hospital care. AMIA Annu Symp Proc 2011; 1428-1435.

34.Pfeifer Vardoulakis L, Karlson A, Morris D, Smith G, Gatewood J, Tan D. Using mobile phones to present medical information to hospital patients. SIGCHI Conf on Human Factors in Computing Systems 2012; May 5: 1411-1420.

35. Pandey A, Hasan S, Dubey D, Sarangi SA. Smartphone apps as a source of cancer information: changing trends in health information-seeking behavior. J Cancer Educ 2013; 28(1): 138-142.

36. Holden RJ, Karsh BT. The technology acceptance model: its past and its future in health care. J Biomed Inform 2010; 43(1): 159-72.

37. MacQueen KM, McLellan E, Kay K, Milstein B. Codebook development for team-based qualitative analysis. Cultural Anthropology Methods 1998; 10(2): 31-36.

38. Creswell JW. Qualitative Inquiry And Research Design: Choosing Among Five Approaches Author: John W. Creswell, Publisher: Sage Publica. 2006, Sage Publications, Inc.

39. Cresswell KM, Bates DW, A Sheikh. Ten key considerations for the successful implementation and adoption of large-scale health information technology. J Am Med Inform 2013; 20(e1): e9-e13.

40. Cresswell K, Sheikh A. Organizational issues in the implementation and adoption of health information technology innovations: an interpretative review. Int J Med Inform 2013; 82(5): e73-e86.

41. Holden RJ, Brown RL, Scanlon MC, Karsh BT. Modeling nurses' acceptance of bar coded medication administration technology at a pediatric hospital. J Am Med Inform Assoc 2012; 19(6): 1050-1058.

42. Carayon P, Li Y, Kelly MM, DuBenske LL, Xie A, McCabe B, Orne J, Cox ED. Stimulated recall methodology for assessing work system barriers and facilitators in family-centered rounds in a pediatric hospital. Appl Ergon 2014; 45(6): 1540-1546.

43.Xie A, Carayon P, Cartmill R, Li Y, Cox ED, Plotkin JA, Kelly MM. Multi-stakeholder collaboration in the redesign of family-centered rounds process. Appl Ergon 2015; 46: 115-123.

44. Kelly MM, Xie A, Carayon P, DuBenske LL, Ehlenbach ML, Cox ED. Strategies for improving family engagement during family-centered rounds. J Hosp Med 2013; 8(4): 201-207.

45. Prey JE, Woollen J, Wilcox L, Sackeim AD, Hripcsak G, Bakken S, Restaino S, Feiner S, Vawdrey DK. Patient engagement in the inpatient setting: a systematic review. J Am Inform Assoc 2014; 21(4): 742-750.

46. Koch SH, Weir C, Westenskow D, Gondan M, Agutter J, Haar M, Liu D, Görges M, Staggers N. Evaluation of the effect of information integration in displays for ICU nurses on situation awareness and task completion time: A prospective randomized controlled study. Int J Med Inform 2013; 82(8): 665-675. 